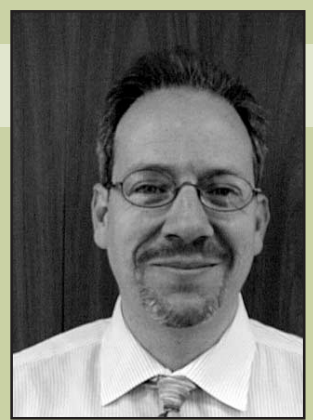

Editor: Richard Doyle Jet Propulsion Lab rdoyle@jpl.nasa.gov

AI in Space

\title{
Model-Based Autonomy in Deep Space Missions
}

\author{
David P. Watson, Johns Hopkins University Applied Physics Laboratory
}

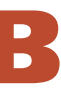

alancing the constrained resources of interplanetary spacecraft with stringent requirements for reliable autonomous operation has been a consistent theme in the evolution of mission systems at the Johns Hopkins Univer-

sity Applied Physics Laboratory. As designer and developer of over 50 spacecraft, APL has addressed this trade-off many times, resulting in many significant scientific and engineering achievements, including landing of the Near Earth Asteroid Rendezvous spacecraft on the asteroid Eros in February 2001 (see Figure 1).

The NEAR mission was one of the first in NASA's Discovery Program. This program's missions are characterized by ambitious scientific goals and highly resourceconstrained systems. Responding to the challenges of operational autonomy in these missions, APL has developed an approach to onboard autonomous decision-making that has served well on multiple NASA missions, in some cases performing critical onboard control in unplanned situations. This article discusses trade-offs involved in current-generation autonomy systems for deep-space applications and describes emerging research that will let future spacecraft operate unattended for significant periods of time, synthesizing control decisions in response to both planned and unplanned events.

\section{Editor's Perspective}

The Johns Hopkins University Applied Physics Lab has recently engaged the challenges of deep-space exploration with some notable successes. The landing on the asteroid Eros to close out the highly successful Near Earth Asteroid Rendezvous mission is one particularly thrilling example. These experiences have led the APL spacecraft and software teams to grapple with challenges such as how to design new, robust fault protection systems for spacecraft as autonomy begins to play a greater role on space missions.

Here, Dave Watson of APL makes the case for the advantages of model-based reasoning approaches. He describes current efforts at APL, in collaboration with Brian Williams' research group at MIT, to develop model-based autonomy capabilities for spacecraft.

\section{The NEAR experience}

The NEAR spacecraft launched in February 1996 with minimal resources for onboard decision-making. Processing on the spacecraft was split between multiple flight computers having approximately 700 Kbytes of available memory for all functions. Despite these limitations, the mission required a variety of autonomous operations of the system, including onboard command validation, closed-loop attitude control in orbit, and most important, spacecraft safing.

This last function, the process of maintaining spacecraft safety in the event of a fault or other unanticipated mission events, is the focus of an ongoing research collaboration between APL and the Massachusetts Institute of Technology Model-Based Embedded Robotic Systems group. Fault management is a particularly critical and complex element of deep-space engineering. Given the potential for extremely large communication latencies in deep-space missions and long periods of flight where no communications are possible, the spacecraft must have sufficient onboard decision-making logic to continuously assess its current situation and issue the appropriate commands to avoid mission failures. Traditionally, systems engineers accomplish this by exhaustively analyzing all potential failure scenarios and developing a minimal set of trigger conditions (a Boolean combination of onboard telemetry values) that activate well-understood response actions.

Figure 2 illustrates the general runtime architecture employed onboard the NEAR spacecraft. Flight system commands issue from two separate processes: command processing and an autonomy engine. Command processing issues time-tagged flight commands that mission operations staff have planned and validated through extensive simulation before uploading them to the flight computer. Once uploaded and validated, command processing simply triggers the flight system commands at their specified time. In the NEAR mission, for example, mission operations staff typically planned, validated, and uploaded a full week at a time of spacecraft operations commands in a continuous pipelined workflow process. These command sequences address all deterministic maneuvering and payload control situations.

Augmenting this "open loop" commanding is the autonomy engine process, which periodically checks the recomputed trigger condition set against real-time telemetry and 
issues response commands if necessary. As Figure 2 shows, ground-based operators can modify trigger conditions, but this occurs much less frequently and shouldn't be required at all if prelaunch systems failure analysis is complete and correct.

This general model of spacecraft autonomy has served well for many missions, restricting the space of potential onboard control actions to enable exhaustive preflight validation, yet providing a minimal degree of reactivity in response to unexpected mission events. Because the approach is also simple, it can be implemented in severely constrained processing architectures. Unfortunately, these benefits come at the cost of severely limited response behaviors. In general, given the difficulty and risk associated with understanding all potential spacecraft subsystem interactions, autonomy command responses are generally limited to placing the spacecraft into a "safe" configuration. This generally consists of powering down all nonessential subsystems and placing the spacecraft in an orientation such that it can receive commands from Earth. In other words, this buys time for human operators to analyze downlinked telemetry and construct response sequences to address the mission anomaly and hopefully restore nominal mission operations.

This time-consuming, labor-intensive process ultimately has become limiting for modern deep-space missions. As the complexity of onboard subsystems grows and we send our spacecraft ever-greater distances, relying on Earth for mission-recovery commands becomes less feasible. The problem facing us now is how to embed autonomy controllers in spacecraft that can address a broad range of mission anomalies with robust, verifiable onboard responses.

\section{Model-based autonomy}

A key milestone in the development of robust onboard autonomy was demonstrated as part of NASA's Deep Space One Probe, which contained a suite of technologies for onboard diagnosis, mission planning, and execution management. ${ }^{1}$ One particular aspect of the DS-1 experiment was model-based autonomy, where the onboard controller was programmed with an abstract, commonsense model of all spacecraft subsystems, which then served to identify and diagnose anomaly conditions in real time. DS-1 limited model- based autonomy to fault identification, but recent research has extended it to form a complete high-level control solution. APL is working with the MIT MERS group to implement a model-based programming environment and onboard executive suitable for use on future resource-constrained deep-space missions.

Our work's foundation is the MIT's prototype model-based execution kernel, known as Titan, and Reactive Model-Based Programming Language (RMPL). ${ }^{2}$ The combination of these tools enables highlevel reasoning about system contingencies, scheduling of commanded operations, inference of a system's hidden state, and control of that state. Figure 3 shows an overview of the Titan model-based executive and its layered relationship with a realtime flight control system. The FCS layer implements low-level command loops, sensor filtering, spacecraft safing logic, and the hardware interface. The Titan-FCS interface is specified in terms of filtered telemetry data and subsystem commands.

Unlike APL's legacy autonomy engine, Titan's deductive controller uses filtered telemetry data ("observations") to infer the state of spacecraft subsystems, then generates a set of control actions to achieve a particular goal state configuration. The sequencer component, executing a high-level control module, provides goal configurations to the deductive controller. Behavior specification to Titan is in the form of activity goals, which ground-based mission planners provide. The executive uses online propositional inference and reactive planning algorithms that reason through complex subsystem interactions to create subsystem commands and specify responses to anomalous mission events. So, the executive operates at longer time scales than the FCS, providing a supervisory level of runtime control.

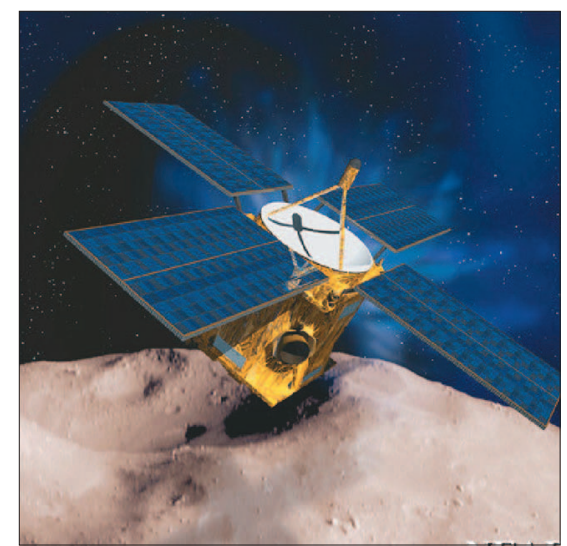

Figure 1. The NEAR spacecraft landing on the asteroid Eros. After a four-year transit and one year of orbital operations, NASA's first Discovery Mission concludes with a landing of the spacecraft on 12 February 2001.

In a way, the Titan executive onboard the spacecraft performs some functions that a ground-based human operations team would handle in current-generation missions. The plant model, coupled with the deductive controller, performs a function analogous to the autonomy engine in our current designs, except that response sequences are not explicitly preprogrammed but synthesized at runtime through iterative diagnosis and reactive replanning. Also, instead of uplinked command sequences, the mission operations team will command the spacecraft with high-level activity goals, which are expressed in terms of synchronous programming constructs and states as defined in the plant model. This approach lets the control sequencer autonomously generate alternative command sequences in response to mission scenarios that might not have been explicitly predicted or understood at system design time.

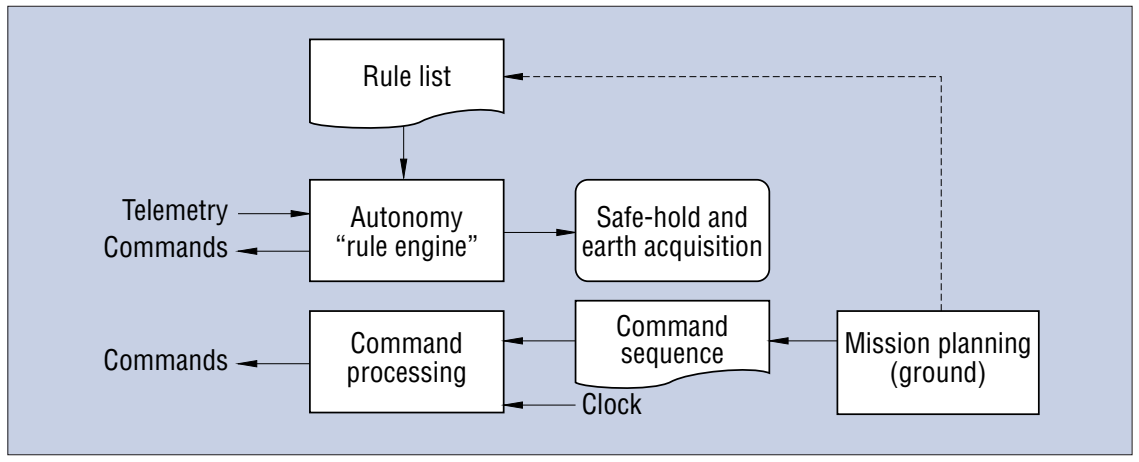

Figure 2. The legacy autonomy architecture employed aboard the NEAR spacecraft. 


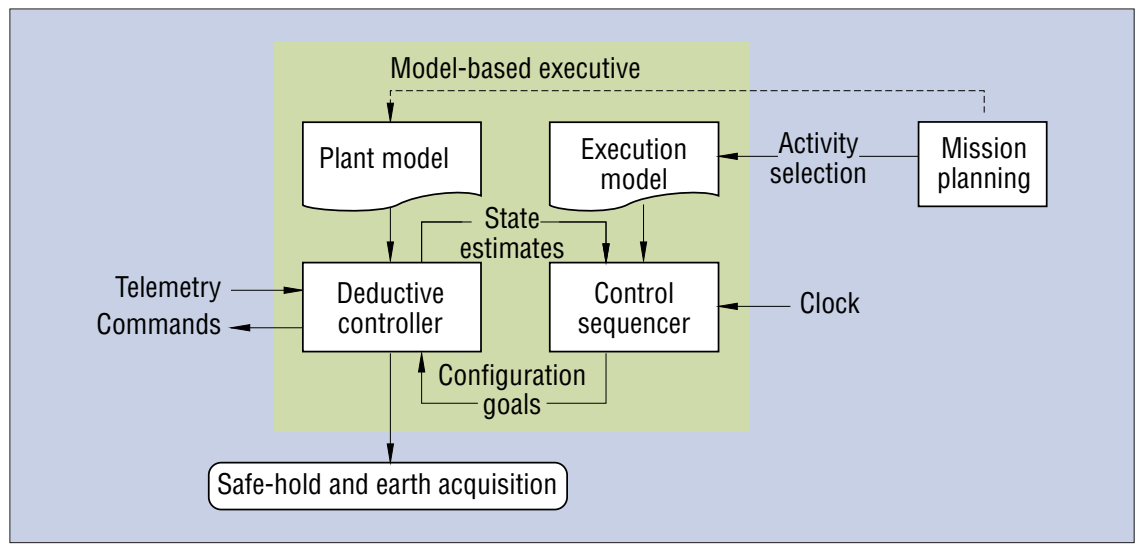

Figure 3. The Titan model-based executive.

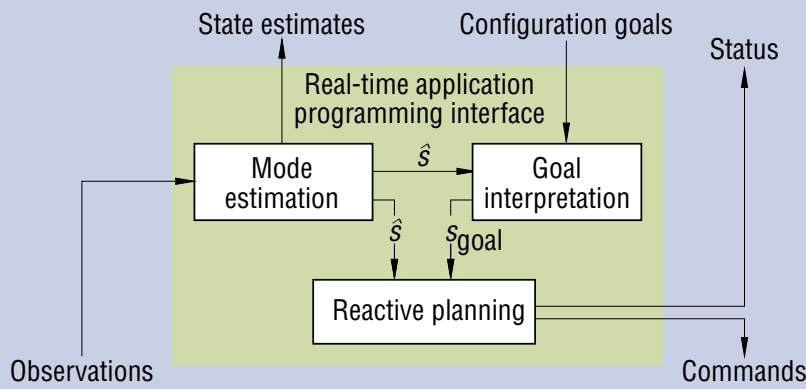

Figure 4. Deductive controller components.

Table 1. Reactive Model-Based Programming Language constructs.

$\begin{array}{ll}\text { Construct } & \text { RMPL syntax } \\ \text { Constraint assertion } & \text { g } \\ \text { Maintenance constraint } & \text { g maintaining m } \\ \text { Conditional execution } & \text { if C thennext A } \\ \text { Guarded transition } & \text { unless C thennext A } \\ \text { Full concurrency } & \text { A, B } \\ \text { Sequential composition } & \text { A; B } \\ \text { Iteration } & \text { always A } \\ \text { Iterated conditional } & \\ \quad \text { execution } & \text { When C donext A } \\ \text { Preemption } & \text { do A watching C } \\ & \end{array}$

\section{Deductive control}

As Figure 3 shows, the deductive controller subdivides into three primary autonomy elements: mode estimation (ME), goal interpretation (GI), and reactive planning (RP). Figure 4 graphically depicts these components and their interactions.

The mode estimation component produces best estimates of the plant state based on observations gathered from the system, knowledge of recent commands issued to the system, constraints defined in the plant mode, and the previous system state. As such, it forms the deductive controller's diagnostic component.

The goal interpretation element identifies the least-cost plant configuration that satisfies some goal state. When provided with the current mode estimate and the desired plant configuration, its companion, the reactive planning component, will produce the next command along some trajectory from Together, GI and RP form the deductivecontroller's mode reconfiguration capability.

The deductive controller's ME and MR elements operate on a single plant model, which is a first-principles encoding of the plant behavior using propositional and limited temporal logic. This shared representation decreases the modeling that the system engineer must perform at design time. Furthermore, this commonality lets the autonomy elements formulate a significant portion of their work as a propositional satisfiability problem and leverage common algorithms for efficiently generating solutions. Propositional models are insufficient, however, for representing the full range of continuous system dynamics that might arise in spacecraft systems control, so researchers are the current state to the desired goal state. extending this work to include hybrid (discrete/continuous) model representations.

As another important component, the deductive controller's real-time application programming interface insulates the discrete event-based autonomy elements from the real-time stream of commands and observations arriving from the underlying plant. The RTAPI enqueues inbound information and dictates the appropriate points in time to perform mode estimation and reconfiguration.

\section{Control sequencing}

Given a particular activity to execute, the control sequencer produces the proper stream of configuration goals for the deductive controller needed to complete the desired activity. Initially, a systems engineer uses RMPL to define these activities in the control model. RMPL provides the representational features of synchronous, reactive languages such as Esterel, Lustre, and Statecharts, with direct interaction with state variables from the plant model. ${ }^{3-5}$ Table 1 summarizes the constructs available in RMPL. The control model is compiled into a finite automata construct known as a hierarchical constraint automata, upon which the control sequencer operates at runtime.

The control sequencer is event driven in that it measures progress through an activity in terms of mode estimation cycles.

Each time the control sequencer receives a mode estimate from the deductive controller, it uses the current state information to mark (activate) and unmark (deactivate) locations in the HCA. The set of marked locations at each cycle defines the configuration goal that will go to the deductive controller along with any maintenance conditions that must be upheld. When an activity is invoked, an initial set of marked locations is produced, and the activity is only completed once all HCA locations have been unmarked or the activity's maximum allotted time has expired.

\section{Operational implications}

The shift to model-based autonomy onboard next-generation spacecraft has several operational implications. The level of abstraction associated with both situation assessment and spacecraft commanding is significantly higher, resulting in requirements for new ground-based tools to support decision support at this higher level. We have just begun to address this prob- 
lem, focusing primarily on diagnosis and situation assessment.

Given the use of declarative, abstract plant models as a basis for onboard control, there is a natural mapping onto analysis displays based on engineering drawings of spacecraft hardware and software subsystems. Figure 5 shows such a display for a model of a spacecraft propulsion system. This interface presents the spacecraft operator a graphic hypothesis of the most likely system state. The operator can query for detailed subsystem state estimates, temporal history, and alternative hypotheses.

Such interface prototypes only hint at the potential for operation of future model-based spacecraft systems. Indeed, we see a potential for model-based analysis at all phases of system design, assisting in conceptual design and trade-off analysis, through reliability and early failure analysis, and into detailed system design and test. Such analysis would thus unify what are currently three separate systems-engineering functions into a single, robust onboard autonomy development framework.

\section{$M$} odel-based programming and execution represents a critical technology for the next generation of autonomous robotic spacecraft. While APL's research focuses on implementing the technology in resource-constrained operational environments, specifically for autonomous fault management, others are developing overall mission frameworks that encompass systems analysis and design requirements as well. The Mission Data System, for example, in development at the NASA Jet Propulsion Laboratory, centers on explicit representation of system state for all planning and control tasks. ${ }^{6}$ Model-based programming and execution technology provides a natural approach to achieving robust fault management in such an architecture, given the similar emphasis on declarative knowledge representation.

APL's next generation of spacecraft systems will operate at significantly greater distances from their operations teams on Earth. The New Horizons mission, for example, will launch in 2006 for a planned rendezvous with Pluto approximately 10 years later. At that point, the spacecraft will be over four billion kilometers from earth, resulting in a round-trip communications time of over

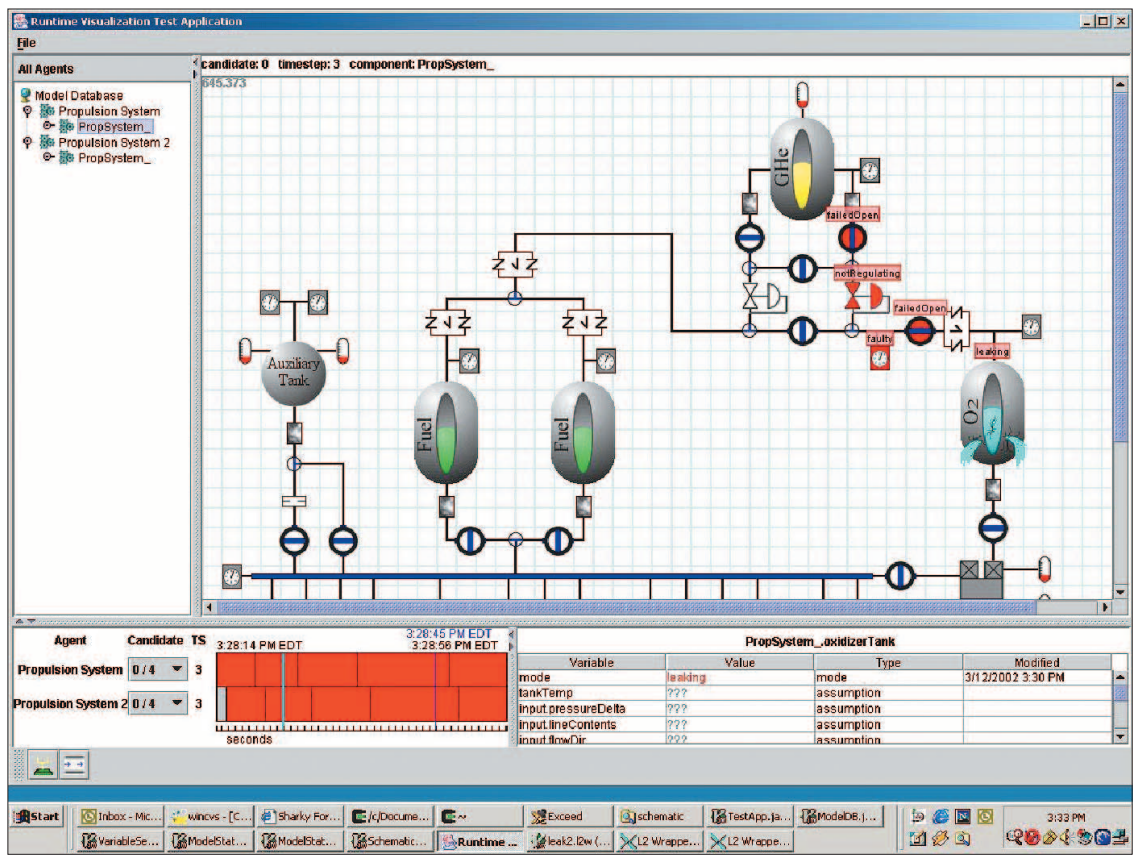

Figure 5. Prototype model-based operations support interface. eight hours. Under such extreme communications latency, the ground operations team will be unable to provide much help in a situation requiring immediate response. Current research in model-based reasoning systems and design frameworks provide an approach to addressing this problem as our future spacecraft continue to grow in complexity, capability, and reach.

\section{Acknowledgments}

This work has been supported in part by the NASA Intelligent Systems Program under contract NAG2-1559. I would also like to thank Brian Williams and Mitch Ingham of MIT, and APL collaborators Mike Pekala and Steve Marshall.

\section{References}

1. D. Bernard et al., "Design of the Remote Agent Experiment for Spacecraft Autonomy," Proc. 1999 IEEE Aerospace Conf., vol. 2,
IEEE Press, 1999, pp. 259-281.

2. B. Williams, "Model-Based Programming of Intelligent Embedded Systems and Robotic Space Explorers," Proc. IEEE, vol. 91, no. 1, Jan. 2003, pp. 212-237.

3. G. Berry and G. Gonthier, "The Esterel Synchronous Programming Language: Design, Semantics, and Implementation," Science of Computer Programming, vol. 19, no. 2, Nov. 1992, pp. 87-152.

4. N. Halbwachs, P. Caspi, and D. Pilaud, "The Synchronous Programming Language Lustre," Proc. IEEE, vol. 79, no. 9, Sept. 1991, pp. 1305-1320.

5. D. Harel, "Statecharts: A Visual Approach to Complex Systems," Science of Computer Programming, vol. 8, no. 3, June 1987, pp. 231-274.

6. D. Dvorak, R. Rasmussen, and T. Starbird; "State Knowledge Representation in the Mission Data System," Proc. IEEE Aerospace Conf., vol. 1, IEEE Press, 2002, pp. 195-204.

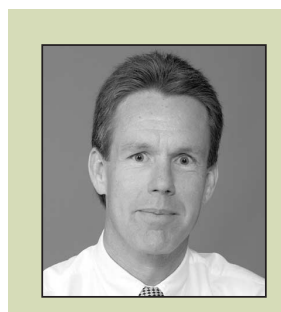

David P. Watson leads the System and Information Sciences Group in the Milton S. Eisenhower Research and Technology Development Center at the Johns Hopkins University Applied Physics Laboratory. Over the past 20 years, he has been involved in the design, development, and evaluation of automated reasoning technologies for simulation, decision support, and system autonomy. He currently leads a collaborative effort with MIT to provide NASA with advanced tools for model-based reasoning in autonomous systems. He holds an MA in applied mathematics from California State University. Reach him at dave.watson@jhuapl.edu. 